
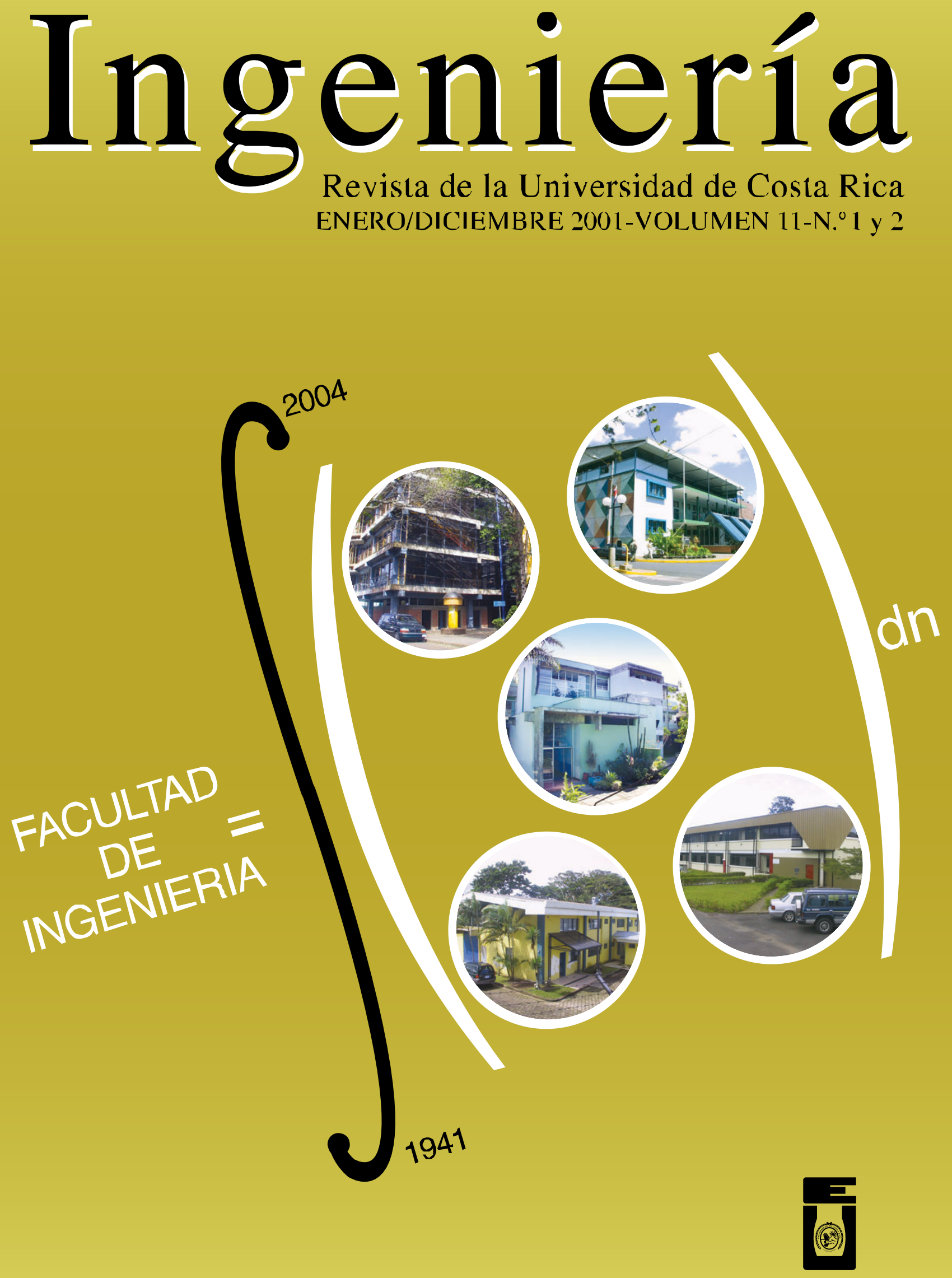


\title{
IDENTIFICACIÓN DE PROCESOS SOBREAMORTIGUADOS UTILIZANDO TÉCNICAS DE LAZO CERRADO
}

Víctor M. Alfaro

\begin{abstract}
Resumen
Se presentan varios de los métodos de identificación de procesos de lazo abierto, disponibles para la obtención de un modelo de primer o segundo orden más tiempo muerto, para un proceso sobreamortiguado.

Mediante pruebas de simulación digital, se compara el desempeño de estos métodos y se determina la bondad de los mismos con base en el error de predicción cometido.

Se dan recomendaciones sobre qué método utilizar con base en el modelo requerido.
\end{abstract}

\begin{abstract}
Several open loop identification methods for over damped process are presented, to obtain first and second order plus dead time models.

A comparison based on the prediction error of the different models is made with the aid of digital simulation in order to measure their performance.

Recommendations on the identification method to use are given based on the model required.
\end{abstract}

\section{INTRODUCCIÓN}

La puesta en servicio de un sistema de control industrial requiere de la correcta sintonización del controlador, es decir de la selección adecuada de sus parámetros.

Para poder sintonizar el controlador $G_{C}(s)$ de un lazo de control, como el mostrado en la Fig. $\mathrm{N}^{\circ} 1$, es necesario identificar primero la dinámica del proceso que se va a controlar $G_{p}(s)$, para luego obtener los parámetros del controlador, empleando el método de sintonización seleccionado. El proceso de sintonización del controlador consta así de dos etapas: identificación y sintonización.

La obtención de la información dinámica del proceso requiere que éste sea excitado de alguna forma y que tanto la entrada aplicada así como la respuesta del proceso, sean registradas. Por estas razones resulta necesario realizar una prueba experimental que permita identificar un modelo dinámico para el proceso.
Las técnicas de identificación experimental, que nos interesan, pueden clasificarse en:

- Métodos basados en la curva de reacción del proceso (respuesta al escalón)

- Métodos de oscilación mantenida

- Métodos de realimentación con relé

- Métodos de control $P$

Los métodos basados en la curva de reacción del proceso son métodos de lazo abierto, el controlador puede o no estar instalado y si lo está operará de modo "manual" durante la prueba.

Los demás métodos son del tipo de lazo cerrado, en donde el controlador se encuentra operando en "automático".

Las técnicas de identificación citadas anteriormente se basan, en consecuencia, en la curva de respuesta del proceso obtenida a lazo abierto o lazo cerrado y son procedimientos esencialmente gráficos. Otros procedimientos que utilizan secuencias 
binarias seudo-aleatorias (PRBS), como señales de prueba y métodos numéricos basados en los mínimos cuadrados para la identificación de modelos en tiempo continuo o en tiempo discreto del tipo ARX, ARMA, ARMAX y otros, están fuera del alcance de este trabajo.
Se presentan a continuación, algunos métodos de identificación de procesos sobreamortiguados basados en la curva de reacción y los resultados comparativos de la bondad de los mismos, obtenidos mediante pruebas de simulación digital.

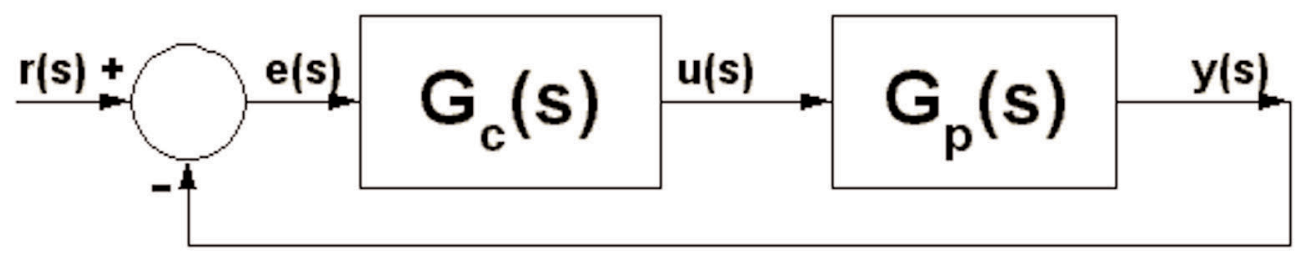

\section{Figura $\mathrm{N}^{0} 1$ - Sistema de control realimentado}

\section{MODELOS}

La mayoría de los métodos de sintonización de controladores se basan en los parámetros de un modelo de orden reducido que permita representar sistemas dinámicos de orden alto y por esta razón los más empleados son los de primer o segundo orden más tiempo muerto, cuyas funciones de transferencia son:

Primer orden más tiempo muerto

$G_{p}(s)=\frac{k_{p} e^{-t_{m} s}}{\tau s+1}$

Segundo orden sobreamortiguado más tiempo тиегto

$$
G_{p}(s)=\frac{k_{p} e^{-t_{m} s}}{\left(\tau_{1} s+1\right)\left(\tau_{2} s+1\right)}
$$

Segundo orden subamortiguado más tiempo muerto

$$
G_{p}(s)=\frac{\omega_{n}^{2} k_{p} e^{-t_{m} s}}{s^{2}+2 \varsigma \omega_{n} s+\omega_{n}^{2}}=\frac{k_{p} e^{-t_{m} s}}{\tau^{2} s^{2}+2 \varsigma \tau s+1}
$$

\section{MÉTODOS DE IDENTIFICACIÓN DE LAZO CERRADO}

A diferencia de los métodos de identificación de lazo abierto -en los cuales se aplica una señal directamente en la entrada de la planta, para identificar los parámetros del modelo deseado a partir de la curva de respuesta de la misma-, los métodos de lazo cerrado deben primero obtener información del comportamiento del sistema bajo alguna condición de control realimentado, y a partir de ésta estimar los parámetros del modelo para la planta.

Para efecto de los métodos de sintonización de controladores, usualmente, se requiere que los sistemas sobreamortiguados de orden superior a uno se representen por medio de un modelo de primer orden más tiempo muerto, como el dado por la ecuación (1); algunos pocos, sin embargo, requieren de un modelo de segundo orden más tiempo muerto, como el dado por (2). Si el sistema es subamortiguado debe representarse por un modelo como el dado por (3).

Restringiremos la presentación de los métodos de identificación, así como los resultados obtenidos en su comparación, a aquéllos que permitan identificar un modelo de primer o segundo orden más tiempo muerto, y en particular a los siguientes:

- Métodos que utilizan la información última (Chen, Ho et al., Lee y Sung)

- Métodos de control $P$ (Jutan y Rodríguez, Lee, Yuwana y Seborg) 


\subsection{MÉTODOS PARA LA OBTENCIÓN DE LA INFORMACIÓN "ÚLTIMA"}

Se denomina información última o crítica a la que se obtiene de la operación del sistema de control en el límite de su estabilidad. Esta información-ganancia última $K_{c u}$ y periodo de oscilación último $T_{u^{-}}$, corresponde a la ganancia del controlador proporcional que lleva el sistema de control al límite de su estabilidad -oscilación mantenida - y al periodo de la oscilación del sistema.

Los valores de los parámetros últimos $K_{c u}, T_{u}$ pueden ser utilizados directamente en un procedimiento de sintonización de controladores o para estimar los parámetros de un modelo para la planta.

\subsubsection{MÉTODO DE OSCILACIÓN MANTENIDA}

Este método fue desarrollado por Ziegler y Nichols[16], como parte de un procedimiento de sintonización de controladores. Con este método se determinan los parámetros $K_{c u}$ y $T_{u}$ a través de un procedimiento iterativo que emplea un controlador básicamente proporcional al que se le han desactivado los modos integral y derivativo. La ganancia del controlador aumenta paulatinamente hasta lograr que la respuesta del sistema a un cambio escalón en el valor deseado sea oscilatoria. El valor de esta ganancia es $K_{c u}$ y el periodo de la respuesta oscilatoria es $T_{u}$.

\subsubsection{MÉTODO DE REALIMENTACIÓN CON RELÉ}

Para obtener la misma información que el método de oscilación mantenida de Ziegler y Nichols, sin necesidad de llevar el sistema al límite de la estabilidad y poder incorporar el procedimiento como parte de un sistema de auto-sintonía del controlador, Aström y Hägglund[2,3] se basaron en el siguiente hecho: un proceso que tenga un retraso de fase de por lo menos $180^{\circ}$ a altas frecuencias, oscilará con un periodo de oscilación igual al periodo crítico bajo el control de un relé. El procedimiento de auto-sintonía incluirá un relé para realizar la prueba de identificación, como se muestra en la Figura $\mathrm{N}^{\circ} 2$. En la Figura $\mathrm{N}^{\circ} 3$ se muestra la respuesta del sistema.

Si la amplitud del relé es $d$, de la expansión en una serie de Fourier se obtiene que la amplitud de la primera armónica de la salida del relé es igual a $4 d / \pi$. Si $a$ es la amplitud de salida del proceso, la ganancia crítica estará dada entonces por:

$$
K_{c u}=\frac{4 d}{\pi a}=\frac{4 d^{\prime}}{\pi a^{\prime}}
$$

y el periodo de oscilación de las señales será el periodo de oscilación último

$$
T_{u}=T_{c}
$$

La amplitud de la oscilación puede cambiarse mediante la variación de la amplitud $d$ del relé.

En los casos en los que el ruido en la señal pueda ocasionar una conmutación intermitente del relé ideal, se debe emplear un relé con histéresis.

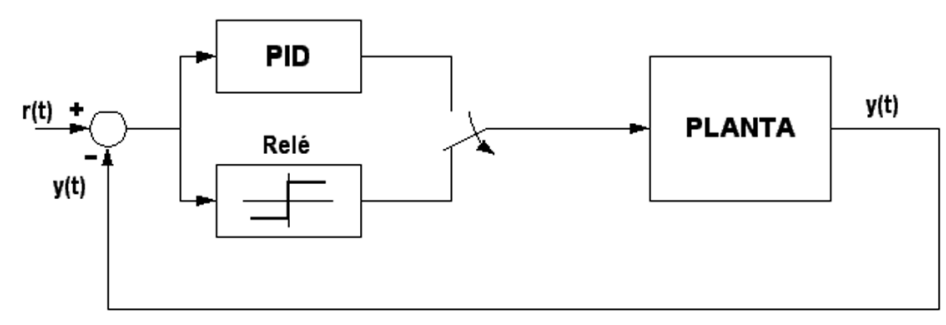

Fig. No 2 - Procedimiento de auto-sintonía de Aström y Hägglund 


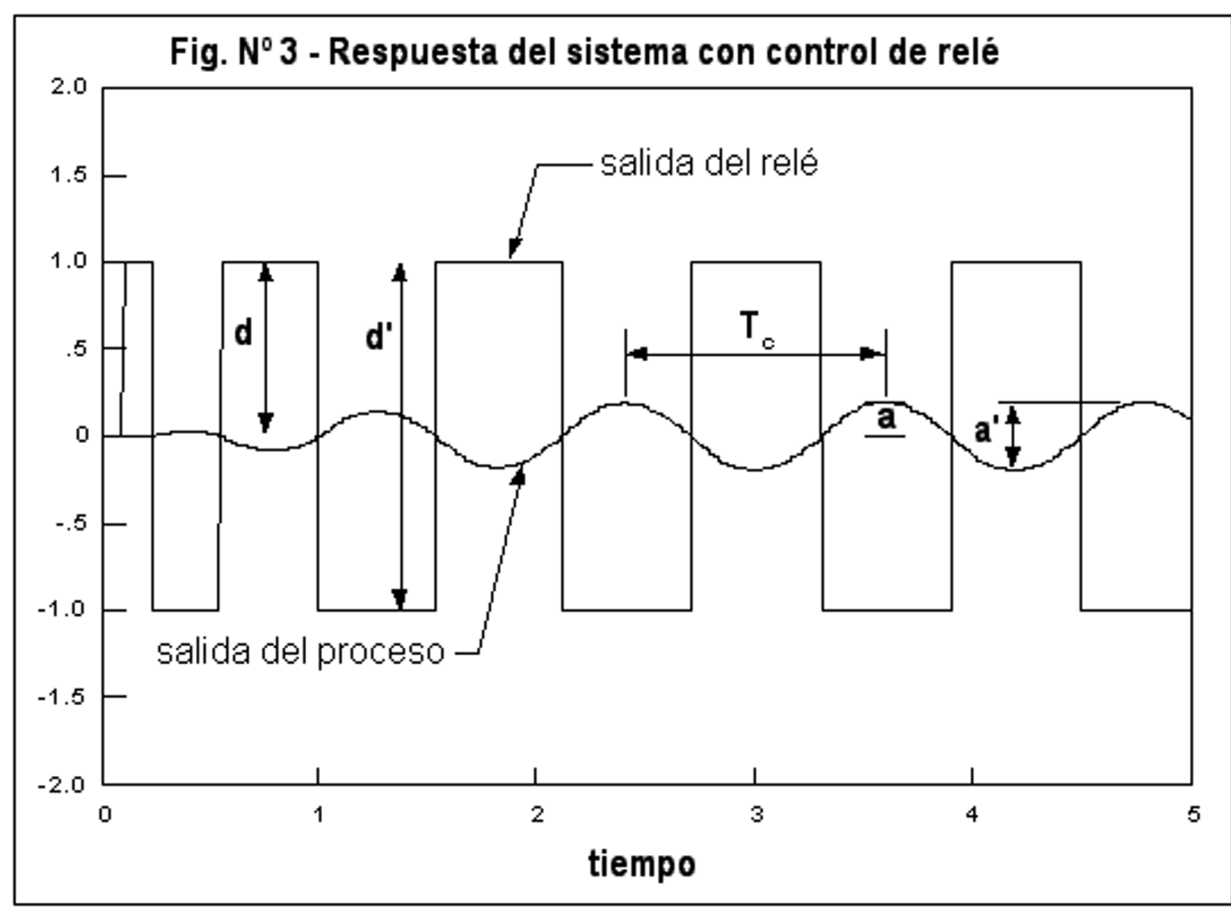

\subsection{MÉTODOS DE IDENTIFICACIÓN BASADOS EN LA INFORMACIÓN ÚLTIMA}

La información última, ya sea obtenida por el método de oscilación mantenida de Ziegler y Nichols o por el de realimentación con relé de Aström y Hägglund, puede emplearse para identificar un modelo para el proceso.

\subsubsection{MÉTODO CHEN[5]}

Este procedimiento combina una prueba de lazo cerrado con un controlador proporcional para determinar la ganancia de la planta, junto con la información última para identificar un modelo de primer orden más tiempo muerto, como el dado por (1) mediante las siguientes ecuaciones:

$k_{p}=\frac{\Delta y}{K_{c}(\Delta u-\Delta y)}$

$$
\begin{aligned}
& \tau=\frac{T_{u}}{2 \pi} \sqrt{K_{c u}^{2} k_{p}^{2}-1} \\
& t_{m}=\frac{T_{u}}{2 \pi}\left[\pi-\tan ^{-1}\left(\frac{2 \pi \tau}{T_{u}}\right)\right]
\end{aligned}
$$

\subsubsection{MÉTODO DE Ho et al.[7]}

Ho utiliza la información última para identificar un modelo con un polo doble más tiempo muerto dado por:

$$
G_{p}(s)=\frac{k_{p} e^{-t_{m} s}}{\left(\tau^{\prime} s+1\right)^{2}}
$$

Mediante las siguientes ecuaciones:

$$
\begin{aligned}
& \tau^{\prime}=\frac{T_{u}}{2 \pi} \sqrt{K_{c u} k_{p}-1} \\
& t_{m}=\frac{T_{u}}{2 \pi}\left[\pi-2 \tan ^{-1}\left(\frac{2 \pi \tau^{\prime}}{T_{u}}\right)\right]
\end{aligned}
$$


En donde la ganancia de la planta se ha obtenido a partir de una prueba de lazo cerrado con un controlador proporcional con (6).

\subsubsection{MÉTODO DE LEE Y SUNG[11]}

Combinando un procedimiento de identificación del relé con una prueba de un controlador proporcional, se obtiene la información necesaria para identificar un modelo de primer orden más tiempo muerto.

Se realiza primero una prueba de retroalimentación con un relé de amplitud $d$ para obtener la amplitud de la salida $a$, y luego una prueba con un controlador $P$ con una ganancia:

$K_{c}=0.6 \frac{4 d}{\pi a}$

El modelo se identifica entonces a partir de:

$$
\begin{aligned}
\tau & =\frac{T_{u} / 2}{\ln \left[\left(1+\frac{a}{k_{p} d}\right) /\left(1-\frac{a}{k_{p} d}\right)\right]} \\
t_{m} & =\tau \ln \left(\frac{1+e^{T_{u} / 2 \tau}}{2}\right)
\end{aligned}
$$

En donde, la ganancia de la planta se ha obtenido de una prueba con control $P$ con (6).

\subsection{MÉTODOS DE CONTROL $P$}

Una de las desventajas del método de identificación de oscilación mantenida de Ziegler y Nichols [3.3.1] radica en la necesidad de llevar el sistema hasta el límite de la estabilidad y puede presentar una operación inestable en presencia de perturbaciones externas. Además, el procedimiento es de prueba y error, por lo tanto, puede llegar a consumir bastante tiempo.

Los métodos basados en la curva de reacción del proceso, tienen la ventaja de requerir una sola prueba, aunque tienen el inconveniente de efectuar ésta en lazo abierto, lo cual significa que no existe ningún control sobre el proceso e inutilización con plantas inestables.

Las características deseables de un método de identificación experimental requieren de una sola prueba en lazo cerrado. Se han desarrollado los métodos de identificación de control $P$, los cuales se basan en la identificación del proceso a partir de la curva de respuesta del sistema a un cambio escalón en el valor deseado, cuando éste se controla con un controlador proporcional: $G_{c}(s)=K_{c}$.

\subsubsection{MÉTODO DE YUWANA Y SEBORG [15]}

Este método fue el primer procedimiento de identificación de lazo cerrado que empleó un controlador proporcional, sin la necesidad de llevar el sistema al límite de la estabilidad. El procedimiento requiere la respuesta del sistema a un cambio escalón en el valor deseado, la cual debe ser subamortiguada. Se modela la misma como la respuesta de un sistema de segundo orden más tiempo muerto, y se identifica para el proceso un modelo de primer orden más tiempo muerto.

De la respuesta del sistema deben obtenerse los siguientes valores: $y_{\mathrm{p} 1}, y_{\mathrm{p} 2}, y_{\mathrm{m} 1}, y_{\mathrm{u}} \mathrm{y} \Delta \mathrm{t}$, correspondientes a los valores del primer y segundo pico de la respuesta, el primer mínimo, el valor final y el semiperiodo, respectivamente, como se muestra en la Fig. $\mathrm{N}^{\circ} 4$.

Suponiendo un modelo de segundo orden más tiempo muerto para el sistema de lazo cerrado, y el empleo de una aproximación de Padé de primer orden para el tiempo muerto dada por:

$$
e^{-t_{m} s}=\frac{1-0,5 t_{m} s}{1+0,5 t_{m} s}
$$

La ganancia del modelo de la planta se puede identificar utilizando (6), y la constante de tiempo y el tiempo muerto aparente de:

$$
K=K_{c} k_{p}
$$




$$
\begin{gathered}
\tau=\frac{\Delta t}{\pi}\left[\varsigma \sqrt{K+1}+\sqrt{\varsigma^{2}(K+1)+K}\right] \sqrt{\left(1-\varsigma^{2}\right)(K+1)} \\
\left.t_{m}=\frac{2 \Delta t \sqrt{\left(1-\varsigma^{2}\right)(K+1)}}{\pi\left[\sqrt{K+1}+\sqrt{\varsigma^{2}(K+1)+K}\right.}\right]
\end{gathered}
$$

Donde: $\varsigma=\left(\varsigma_{1}+\varsigma_{2}\right) / 2$

$$
\begin{gathered}
\boldsymbol{\varsigma}_{1}=\frac{-\ln \left[\left(y_{u}-y_{m 1}\right) /\left(y_{p 1}-y_{u}\right)\right\rfloor}{\sqrt{\pi^{2}+\ln ^{2}\left[\left(y_{u}-y_{m 1}\right) /\left(y_{p 1}-y_{u}\right)\right]}} \\
\boldsymbol{\varsigma}_{2}=\frac{-\ln \left[\left(y_{p 2}-y_{u}\right) /\left(y_{p 1}-y_{u}\right)\right]}{\sqrt{4 \pi^{2}+\ln ^{2}\left[\left(y_{p 2}-y_{u}\right) /\left(y_{p 1}-y_{u}\right)\right]}}
\end{gathered}
$$

\subsubsection{MÉTODO DE JUTAN Y RODRÍGUEZ[9]}

Yuwana y Seborg [3.3.1] utilizaron en su método de identificación una aproximación de Padé de primer orden para el tiempo muerto, lo cual introduce errores en el proceso de identificación. En vez de ésta, Jutan y Rodríguez proponen utilizar la siguiente aproximación:

$e^{-t_{m} s}=\frac{1+\gamma_{1} t_{m} s+\gamma_{2} t_{m}^{2} s^{2}}{1+\delta t_{m} s}$

$\operatorname{con} \gamma_{1}=-0,61453, \gamma_{2}=0,1247$ y $\delta=0,3866$.

La ganancia del modelo se obtiene de (6) y los otros parámetros de:

$$
\begin{aligned}
& \tau=\frac{-b \pm\left(b^{2}-4 a c\right)^{1 / 2}}{2 a} \\
& t_{m}=\alpha+\beta \tau
\end{aligned}
$$

Donde:

$$
\begin{aligned}
a & =\beta^{2} \gamma_{2} K+\beta \delta \\
b & =2 \gamma_{2} K \alpha \beta+\alpha \delta \\
c & =\gamma_{2} K \alpha^{2}-\tau^{\prime 2}(1+K) \\
\alpha & =\frac{2 \varsigma^{\prime} \tau^{\prime}(1+K)}{\delta+\gamma_{1} K} \\
\beta & =-\left(\delta+\gamma_{1} K\right)^{-1} \\
K & =\frac{\Delta y}{\Delta u-\Delta y} \\
\varsigma^{\prime} & =\frac{-\ln \alpha_{1}}{\left[\tau^{2}+\ln ^{2} \alpha_{1}\right]^{\prime 2}} \\
\alpha_{1} & =\frac{y_{u}-y_{m 1}}{y_{p 1}-y_{u}} \\
\tau^{\prime}= & \frac{\Delta t}{\pi} \sqrt{1-\varsigma^{\prime 2}}
\end{aligned}
$$

\subsubsection{MÉTODO DE LEE[10]}

A diferencia de Yuwana y Seborg [3.3.1] y de Jutan y Rodríguez [3.3.2], ambos utilizan aproximaciones para sustituir el tiempo muerto que aparece en el denominador de la función de transferencia de lazo cerrado. Lee logra que los polos de la función de transferencia, identificada para la respuesta subamortiguada con control $P$, coincidan con los de la desarrollada a partir del supuesto de que la planta controlada sea de primer orden más tiempo muerto.

La ganancia se obtiene con (6) y la constante de tiempo y el tiempo muerto de:

$$
\begin{gathered}
e^{-\alpha t_{m} s}-\frac{K_{c} k_{p}}{\beta}\left[\alpha \operatorname{sen}\left(\beta t_{m}\right)-\beta \cos \left(\beta t_{m}\right)\right]=0 \\
\tau=\frac{1}{\alpha}\left[1+K_{c} k_{p} e^{\alpha t_{m}} \cos \left(\beta t_{m}\right)\right]
\end{gathered}
$$

Donde $\alpha=\varsigma / \tau^{\prime} \quad$ y $\beta=\sqrt{1-\varsigma^{2}} / \tau^{\prime}$ 
La ecuación (27) es no lineal y debe resolverse en forma iterativa utilizando el siguiente algoritmo:

$t_{m, k+1}=\frac{1}{\beta}\left[v+\tan ^{-1}(\Psi)\right]$

$\Psi=\frac{\beta e^{-\alpha t_{m k}}}{K_{c} k_{p} \sqrt{\alpha^{2}+\beta^{2}} \cos \left(\beta t_{m . k}-v\right)}$

$v=\tan ^{-1}(\beta / \alpha)$
Iniciándolo con:

$t_{m, 0}=(v+\pi / 4) / \beta$

Una vez que (30) converja, el valor obtenido del tiempo muerto $t_{m}$ se utiliza para calcular la constante de tiempo $\tau$ con (28).

\section{Fig. $\mathbf{N}^{0} \mathbf{4}$ - Respuesta del sistema con control P}

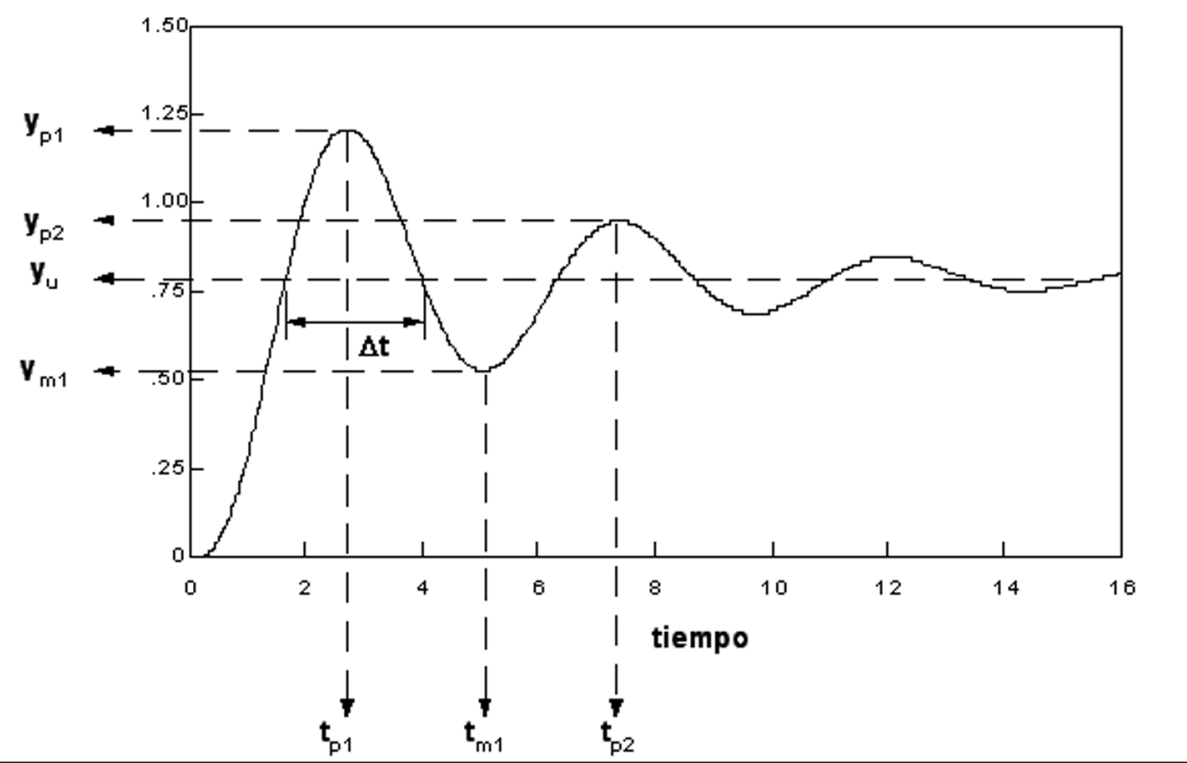

\section{PRUEBAS COMPARATIVAS POR SIMULACIÓN}

La bondad de las técnicas de identificación descritas anteriormente se evaluó mediante la simulación digital, tanto de las plantas de prueba como de los modelos identificados, para ello se utilizó el programa VisSim 3.0[13].

\subsection{PLANTAS DE PRUEBA}

La selección de las plantas empleadas para las pruebas comparativas se basó en las plantas utili- zadas para tal fin en otras investigaciones[4,6,7,8,12,14], así como en variantes de las mismas. Estas son

$$
\begin{aligned}
& G_{p 1}(s)=\frac{1}{(\tau s+1)^{3}}, \tau=0.5,1.0,1.5,2.0 \\
& G_{p 2}(s)=\frac{1}{(0.2 \mathrm{~s}+1)(0,5 \mathrm{~s}+1)(\mathrm{s}+1)} \\
& (32) \\
& G_{p 3}(s)=\frac{1}{(0,1 s+1)(0,2 s+1)(0,5 s+1)(s+1)(2 s+1)}
\end{aligned}
$$




$$
G_{p 4}(s)=\frac{e^{-.15 s}}{(0,33 \mathrm{~s}+1)(0,5+1)(\mathrm{s}+1)}
$$

\section{2 ÍNDICES DE DESEMPEÑO}

Definiendo el "error de predicción" de un modelo como la diferencia entre la salida de la planta y la predicha por éste, se establecieron los siguientes índices de desempeño:

Integral del error de predicción absoluto

$$
I E A P=\int_{0}^{\infty}\left|y_{p}(t)-y_{m}(t)\right| d t
$$

Integral del error de predicción cuadrático

$$
I E C P=\int_{0}^{\infty}\left(y_{p}(t)-y_{m}(t)\right)^{2} d t
$$

En donde $y_{p}(t)$ es la salida de la planta, y $y_{m}(t)$ la del modelo identificado para la misma.

El índice IEAP representa el área diferencial entre la respuesta de la planta y la del modelo, de manera que si la IEAP $\rightarrow 0$ entonces $y_{m}(t) \rightarrow$ $y_{p}(t)$.

Cuanto menor sea este índice de desempeño mejor será la representación dada por el modelo.

El índice IECP da mayor peso a las desviaciones grandes que a las pequeñas, por ello considerando dos modelos que posean valores del IEAP similares, el que posea un menor IECP predecirá la salida de la planta con desviaciones máximas menores.

Como la evaluación hasta el infinito de los índices (35) y (36) es imposible de realizar, éstos se evaluarán para un intervalo de tiempo

entre 0 y $10 \tau_{T}$, siendo $\tau_{T}=\sum_{i=1}^{n} \tau_{i} \quad$ el

sumatorio de las constantes de tiempo de la planta.

\subsection{PARÁMETROS ÓPTIMOS DE LOS MODELOS}

Los índices de desempeño definidos en [4.2] permiten realizar una comparación entre los diferentes modelos identificados para una planta en particular, pero más importante que su cuantificación para un modelo dado, es su valor relativo respecto al de otro.

Para establecer un valor o índice de referencia que compare la bondad de los modelos, se identificaron los parámetros del modelo de primer y segundo orden más tiempo muerto que minimizan el criterio IEAP, y se definió el porcentaje de error de predicción como:

$$
P E P_{i}=100 \frac{I E A P_{i}-I E A P_{o p t}}{I E A P_{o p t}}
$$

En donde $I E A P_{\mathrm{i}}$ es el valor del índice $I E A P$ para un procedimiento de identificación en particular, y el $I E A P_{\text {opt }}$ el del modelo optimizado para esa misma planta.

\subsection{RESULTADOS DE LA SIMULACIÓN}

Las cuatro plantas descritas en [4.1] se identificaron con los métodos enumerados en [3.0] y su desempeño fue evaluado por medio de los índices establecidos en [4.2].

Para el caso particular de la planta $G p 3$, en la Tabla $\mathrm{N}^{\mathrm{o}} 1$ se muestran los modelos óptimos de primer y segundo orden más tiempo muerto identificados y utilizados para medir la bondad de los métodos de identificación comparados; en la Tabla $\mathrm{N}^{\circ} 2$ se indican los parámetros últimos obtenidos con esta planta por el método de oscilación mantenida y por el de realimentación con relé; en la Tabla $\mathrm{N}^{\mathrm{o}} 3$ los modelos obtenidos a partir de esta información y en la Tabla $\mathrm{N}^{\circ} 4$ los modelos obtenidos con las técnicas de control $P$. 
Tabla $N^{\circ} 1$ - Parámetros de los modelos óptimos respecto a la IEAP

\begin{tabular}{|c|c|c|c|c|c|c|c|c|}
\hline Modelo & $\boldsymbol{k}_{\mathrm{p}}$ & $\boldsymbol{t}_{\mathrm{m}}$ & $\boldsymbol{\tau}_{\mathbf{1}}$ & $\tau_{\mathbf{2}}$ & $\zeta$ & $\omega_{\mathrm{n}}$ & IEAP* $^{*}$ & IECP* $^{*}$ \\
\hline Primer orden & 1 & $\begin{array}{c}2.309 \\
9\end{array}$ & $\begin{array}{c}1.598 \\
0\end{array}$ & - & - & - & $\begin{array}{c}1.849 \mathrm{E} \\
-1\end{array}$ & $\begin{array}{c}9.026 \mathrm{E} \\
-3\end{array}$ \\
\hline $\begin{array}{c}\text { Segundo } \\
\text { orden }\end{array}$ & 1 & $\begin{array}{c}0.627 \\
0\end{array}$ & $\begin{array}{c}1.600 \\
0\end{array}$ & $\begin{array}{c}1.597 \\
0\end{array}$ & 1.0000 & 0.6256 & $\begin{array}{c}3.896 \mathrm{E} \\
-2\end{array}$ & $\begin{array}{c}1.992 \mathrm{E} \\
-4\end{array}$ \\
\hline
\end{tabular}

* en el intervalo de solución de 0 a 38

\begin{tabular}{|c|c|c|c|}
\hline \multicolumn{3}{|c|}{ Tabla $N^{\boldsymbol{0}} 2$ - Información "última” } \\
\hline \multicolumn{2}{|c|}{ Ziegler y Nichols } & \multicolumn{2}{c|}{ Aström y Hägglund (relé) } \\
\hline$K_{c u}$ & $T_{u}$ & $K_{c u}$ & $T_{u}$ \\
\hline 6.100 & 4.660 & 5.940 & 4.690 \\
\hline
\end{tabular}

\begin{tabular}{|c|c|c|c|c|c|c|c|}
\hline \multicolumn{7}{|c|}{ Tabla $N^{*}$ 3 - Modelos basados en la información "última" } \\
\hline \multicolumn{7}{|c|}{ Modelo de primer orden más tiempo muerto - Método de Chen } \\
\hline $\boldsymbol{k}_{\boldsymbol{p}}$ & $\boldsymbol{t}_{\boldsymbol{m}}$ & $\tau$ & $\boldsymbol{n}$ & IEAP* & IECP* & PEP \\
\hline $1(1)$ & 1.2871 & 4.4629 & 1 & $1.946 \mathrm{E}+0$ & $2.576 \mathrm{E}-1$ & 952 \\
\hline $1(2)$ & 1.2988 & 4.3706 & 1 & $1.866 \mathrm{E}+0$ & $2.391 \mathrm{E}-1$ & 909 \\
\hline \multicolumn{7}{|c|}{ Modelo de primer orden más tiempo muerto - Método de Lee y Sung } \\
\hline $\boldsymbol{k}_{\boldsymbol{p}}$ & $\boldsymbol{t}_{\boldsymbol{m}}$ & $\tau$ & $\boldsymbol{n}$ & IEAP* & IECP* & PEP \\
\hline $1(2)$ & 1.2991 & 5.3852 & 1 & $2.876 \mathrm{E}-1$ & $5.090 \mathrm{E}-1$ & 1,455 \\
\hline \multicolumn{7}{|c|}{ Modelo de polo doble más tiempo muerto - Método de Ho et al. } & \\
\hline $\boldsymbol{k}_{\boldsymbol{p}}$ & $\boldsymbol{t}_{\boldsymbol{m}}$ & $\tau$ & $\boldsymbol{n}$ & IEAP & IECP* & PEP** \\
\hline $1(1)$ & 0.6183 & 1.6749 & 2 & $1.658 \mathrm{E}-1$ & $3.322 \mathrm{E}-3$ & 326 \\
\hline $1(2)$ & 0.6312 & 1.6590 & 2 & $1.475 \mathrm{E}-1$ & $2.684 \mathrm{E}-3$ & 279 \\
\hline
\end{tabular}

* en el intervalo de solución de 0 a 38

** respecto a la IAEP del modelo óptimo de segundo orden sobreamortiguado

(1) Se emplea la información última de Ziegler y Nichols

(2) Se emplea la información última de la prueba con relé de Aström y Hägglund 


\subsection{ANÁLISIS DE LOS RESULTADOS}

El análisis y la comparación del desempeño de los métodos de identificación estudiados, consideran los resultados obtenidos con las cuatro plantas de prueba.

\subsubsection{MÉTODOS PARA LA OBTENCIÓN DE LA INFORMACIÓN ÚLTIMA}

Como se puede apreciar en la Tabla $\mathrm{N}^{\circ}$ 2, para la planta $G_{p 3}$, los parámetros últimos obtenidos por ambos procedimientos son prácticamente los mismos. En el valor del periodo de oscilación la diferencia es despreciable y en el caso de la ganancia, el error cometido al emplear el método del relé fue poco más del $2 \%$.

Las pruebas demostraron que ambos procedimientos identifican casi en forma idéntica el periodo de oscilación, y que el error máximo en la estimación de la ganancia última cometido con el método de realimentación con relé fue del orden del $4 \%$.

\begin{tabular}{|c|c|c|c|c|c|c|}
\hline \multicolumn{7}{|c|}{ Tabla $N^{\circ} 4-$ Métodos de control $P$} \\
\hline \multicolumn{7}{|c|}{ Modelos de primer orden más tiempo muerto } \\
\hline \multicolumn{7}{|c|}{ Yuwana y Seborg } \\
\hline$K_{c}$ & $k_{p}$ & $T_{m}$ & $\tau$ & $I A E P^{*}$ & $I S E P^{*}$ & PEP \\
\hline 2 & 1 & 1.6880 & 3.5280 & $1.413 \mathrm{E}+0$ & $1.549 \mathrm{E}-1$ & 664 \\
\hline 3 & 1 & 1.6930 & 3.8880 & $1.778 \mathrm{E}+0$ & $2.341 \mathrm{E}-1$ & 862 \\
\hline 4 & 1 & 1.6850 & 4.2410 & $2.122 \mathrm{E}+0$ & $3.189 \mathrm{E}-1$ & 1,048 \\
\hline 5 & 1 & 1.6700 & 4.5800 & $2.446 \mathrm{E}+0$ & $4.060 \mathrm{E}-1$ & 1,223 \\
\hline \multicolumn{7}{|c|}{ Jutan y Rodríguez } \\
\hline$K_{c}$ & $k_{p}$ & $T_{m}$ & $\tau$ & $I A E P^{*}$ & $I S E P^{*}$ & PEP \\
\hline 2 & 1 & 1.7560 & 3.2710 & $1.227 \mathrm{E}+0$ & $1.237 \mathrm{E}-1$ & 564 \\
\hline 3 & 1 & 1.6220 & 3.6870 & $1.506 \mathrm{E}+0$ & $1.705 \mathrm{E}-1$ & 715 \\
\hline 4 & 1 & 1.5370 & 4.0360 & $1.770 \mathrm{E}+0$ & $2.242 \mathrm{E}-1$ & 857 \\
\hline 5 & 1 & 1.4720 & 4.3460 & $2.014 \mathrm{E}+0$ & 2.797E-1 & 989 \\
\hline \multicolumn{7}{|l|}{ Lee } \\
\hline$K_{c}$ & $K_{p}$ & $T_{m}$ & $\tau$ & $I A E P^{*}$ & $I S E P^{*}$ & PEP \\
\hline 2 & 1 & 1.6388 & 3.4138 & $1.250 \mathrm{E}+0$ & $1.219 \mathrm{E}-1$ & 576 \\
\hline 3 & 1 & 1.5076 & 3.7161 & $1.421 \mathrm{E}+0$ & $1.491 \mathrm{E}-1$ & 669 \\
\hline 4 & 1 & 1.4287 & 4.0052 & $1.631 \mathrm{E}+0$ & $1.898 \mathrm{E}-1$ & 782 \\
\hline 5 & 1 & 1.3719 & 4.2350 & $1.803 \mathrm{E}+0$ & $2.264 \mathrm{E}-1$ & 875 \\
\hline
\end{tabular}

* en el intervalo de solución de 0 a 38 


\subsubsection{MÉTODOS BASADOS EN LA INFORMACIÓN ÚLTIMA}

Para identificar un modelo de primer orden más tiempo muerto se utilizó el método de Chen, y el de Ho et al. para identificar uno con un polo doble.

En cada caso se identificaron dos modelos, uno basado en la información obtenida por el procedimiento de Ziegler y Nichols, y el otro por la información del relé de Aström y Hägglund.

En el caso del método de Chen, el PEP de ambos modelos fue muy alto y del mismo orden de magnitud, desde $740 \%$ hasta más de $1900 \%$, con una ligera ventaja en el identificado a partir de la información obtenida por el método del relé.

El modelo de polo doble de Ho mostró ser superior al de Chen con valores del PEP entre el 235 $\%$ y el $630 \%$ al compararse con el modelo óptimo de segundo orden, siendo el modelo identificado a partir de la información del relé ligeramente mejor en todos los casos. Es importante destacar que si se compara con el modelo óptimo de primer orden, el modelo de polo doble de Ho fue inferior en el caso de las dos primeras plantas y ligeramente superior en el caso de las otras dos.

El modelo identificado por el método de Lee y Sung, que los autores proponen emplear a partir de la información última obtenida por el método del relé, tuvo el peor desempeño entre todos los métodos de lazo cerrado probados, con valores del PEP entre $1200 \%$ y $2620 \%$.

\subsubsection{MÉTODOS DE CONTROL $P$}

Los métodos de Jutan y Rodríguez y el de Lee fueron propuestos para mejorar los de Yuwana y Seborg; los resultados muestran que los porcentajes de error de predicción son en promedio entre un $5 \%$ y un $18 \%$ menores en el caso de Jutan y Rodríguez, y entre un $4 \%$ y un 24 $\%$ en el caso de Lee. Es importante notar que basándose en los promedios del PEP, el método de Lee resulta ser mejor que el de Jutan y Rodríguez aunque éste último es superior al de Lee para los valores bajos de la ganancia del controlador, deteriorándose a mayor velocidad con el aumento de la ganancia.

En general, los métodos de control $P$ mostraron índices de PEP altos, entre $530 \%$ y $1900 \%$, según el método empleado y la ganancia del controlador utilizada en la prueba.

En la Fig. $\mathrm{N}^{\circ} 5$ se muestra la curva de reacción del proceso junto con la respuesta de los modelos identificados por los métodos de Chen, Jutan y Rodríguez y de Ho (polo doble). 


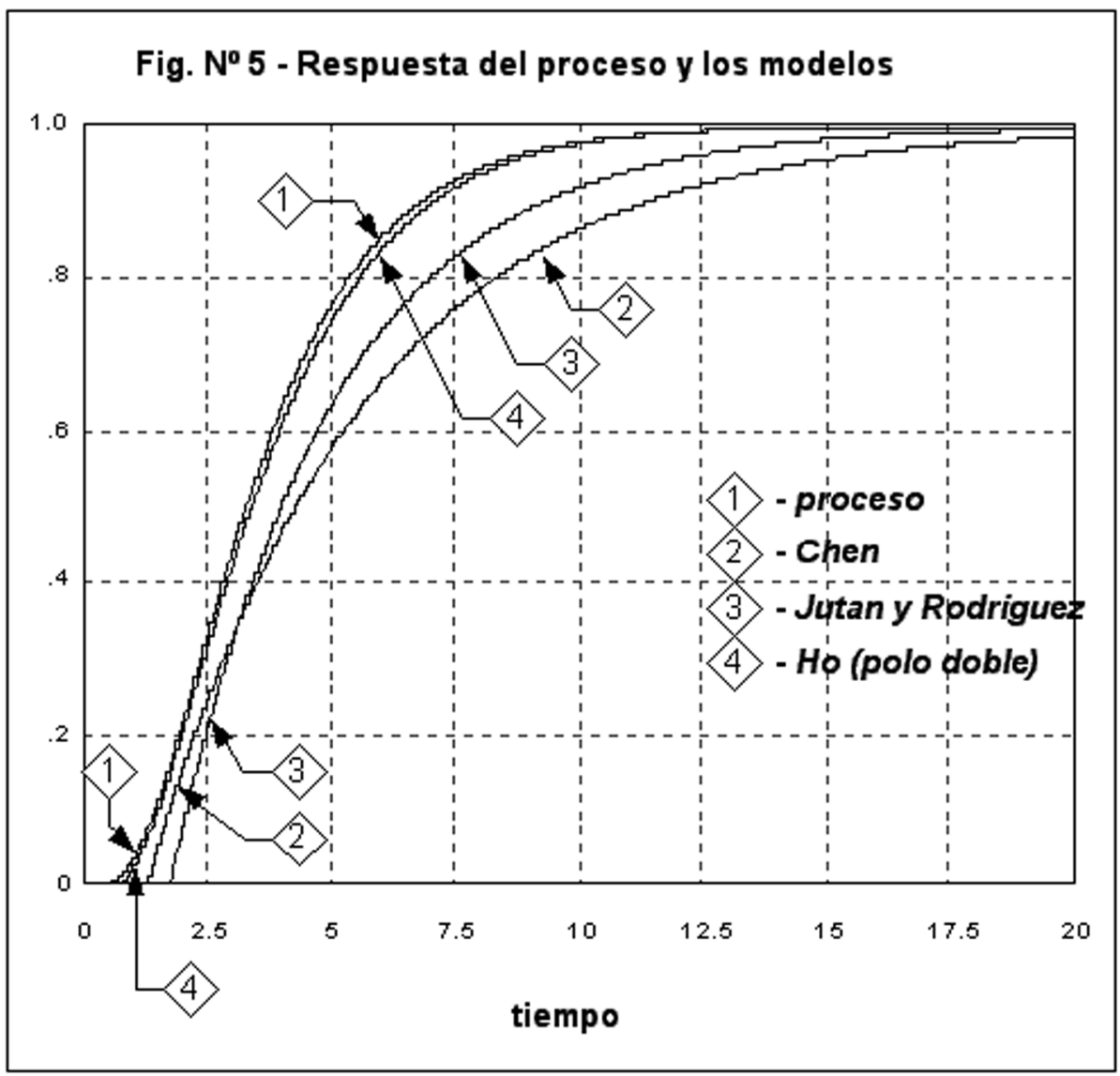

\section{CONCLUSIONES}

Los métodos de sintonización de controladores parten de un modelo identificado para el proceso a controlar; por lo tanto, la fidelidad con que el modelo represente su dinámica es de primordial importancia para lograr los objetivos de control deseados.

Se considera que la diferencia entre los valores obtenidos para la información última $K_{c u}$ y $T_{u}$ por el método de oscilación mantenida de Ziegler y Nichols y el del relé de Aström y Hägglund no es significativa, con la ventaja de que el método del relé no requiere llevar el sistema al límite de la estabilidad. Este procedimiento de identificación ha sido incorporado dentro de varios controladores PID comerciales.
Aunque la información última se obtiene con bastante exactitud mediante cualquiera de los dos procedimientos anteriores, los modelos identificados a partir de ésta por Chen, Ho y Lee mostraron resultados inferiores a los procedimientos de identificación de lazo abierto, por el hecho de que sistemas con comportamientos oscilatorios a lazo cerrado similares pueden tener respuestas transitorias de lazo abierto bastante diferentes.

Para los métodos con control $P$, los resultados muestran claramente que la ganancia del controlador incide sobre los resultados obtenidos, a mayor ganancia mayor es el porcentaje de error de predicción. En conclusión, en estos métodos deberá utilizarse la menor ganancia del controlador que produzca una respuesta subamortiguada, en la 
cual se puedan identificar por lo menos los dos primeros picos, y emplear el método de Jutan y Rodríguez para la identificación, por no requerir resolver una ecuación no lineal por medios iterativos.

Los métodos de control $P$ incluyen dos aproximaciones en el procedimiento de identificación, primero se aproxima el sistema de lazo cerrado por un modelo de segundo orden subamortiguado más tiempo muerto y luego, utilizando una aproximación de Padé u otra similar para el tiempo muerto, se deriva el modelo del proceso. La acumulación de los errores introducidos por estas dos aproximaciones les hace tener un desempeño inferior al de los métodos de lazo abierto.

Si se comparan los valores de los PEP de los métodos de identificación de lazo cerrado probados aquí, con los valores de los métodos basados en la curva de reacción del proceso comparados por Alfaro[1] con antelación, es evidente que estos últimos son superiores, ya que en general tienen valores del PEP mucho menores. Por ejemplo, los modelos de primer orden más tiempo muerto identificados con los métodos de dos puntos (Ho, Alfaro, Chen y Yang) tuvieron un PEP entre el $3 \%$ y el $19 \%$ solamente, en comparación con el PEP de $900 \%$ del mejor modelo de primer orden identificado con los procedimientos de lazo cerrado.

Los resultados permiten recomendar que se emplee un método de identificación de lazo abierto y que en los casos en que esto no sea posible, se utilice el método de Chen para identificar un modelo de primer orden más tiempo muerto o el de Ho para uno de segundo orden más tiempo muerto, a partir de la información última obtenida de la prueba del relé.

\section{BIBLIOGRAFÍA}

1. Alfaro, V.M. - "Identificación de procesos sobreamortiguados utilizando técnicas de lazo abierto", Ingeniería (Costa Rica), Vol. $11 \mathrm{~N}^{\circ} 2,2001$.
2. Aström, K.J. y T. Hägglund - “Automatic Tuning of Simple Regulators with Specifications on Phase and Amplitude Margins", Automatica (UK), Vol. $20 \mathrm{~N}^{\circ}$ 5, pág. 645-651, 1984.

3. Aström, K.J. y T. Hägglund - “Automatic Tuning of PID Controllers", Research Triangle Park, NC. EUA, Instrument Society of America, 1988.

4. Aström, K.J. y T. Hägglund - "Benchmark Systems for PID Control", IFAC Workshop on Digital Control: Past, Present and Future of PID Control, Terrassa, España, Abril 5 - 7, 2000.

5. Chen, C.L. - "A Simple Method for On-Line Identification and Controller Tuning", AIChE Journal (EUA), Vol. 35 N 12, Dic. 1989.

6. Chen, C.L. y S.F. Yang - "PI tuning based on peak amplitude ratio", Preprints of IFAC Workshop on Digital Control: Past, Present and Future of PID Control, Terrassa, España, Abril 5 - 7, 2000.

7. Ho, W.K., C.C. Hang y L.S. Cao - "Tuning PID Controllers Based on Gain and Phase Margin specifications", Automatica (UK),

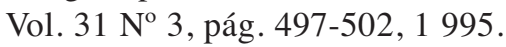

8. Jahanmiri, A. y H.R. Fallahi - "New Methods for Process Identification and design of Feedback Controllers", Transactions of The Chemical Engineers (EUA), Vol. 75 Part A, Jul. 1997.

9. Jutan, A. y E.S. Rodríguez - "Extension of a New Method for On-Line Controller Tuning", The Canadian Journal of Chemical Engineers (Canada), Vol. 62, Dic. 1984.

10. Lee, J. - "On-Line PID Controller Tuning from a Single, Close -Loop Test", AIChE Journal (EUA), Vol. 35 N 2, Feb. 1989. 
11. Lee, J. y S.W. Sung - "Comparison of Two Identification Methods for PID Controller tuning", AIChE Journal (EUA), Vol. $39 \mathrm{~N}^{\circ}$ 4, Abril 1993.

12. Nishikawa, Y., N. Sannomiya, T. Ohta y H. Tanaka - "A Method for Auto-Tuning of PID Controller Parameters", Automatica (UK), Vol. 20 N 3, pág. 321-332, 1984.

13. Visual Solutions - "VisSim User's Guide Version 3", Westford, Ma., EUA, Visual Solutions, 1999.

14. Vitecková, M., A. Viecek y L. Smitny "Simple PI and PID Controllers tuning for monotone self regulation plant", IFAC Workshop on Digital Control: Past, Present and Future of PID Control, Terrassa, España, Abril 5 - 7, 2000.

15. Yuwana, M. y D.E. Seborg - "A New Method for On-Line Controller Tuning", AIChE Journal (EUA), Vol. $23 \mathrm{~N}^{\circ}$ 3, Mayo 1982.

16. Ziegler, J.B. y N.B. Nichols - Optimum Settings for Automatic Controls", ASME Transactions (EUA), Vol. 64, pág. 759768, 1942.

\section{SIMBOLOGÍA}

$G_{p}(s) \quad$ función de transferencia (modelo)

$G_{c}(s) \quad$ función de transferencia (controlador)

$k_{p} \quad$ ganancia estática

$\tau, \tau_{1}, \tau_{2} \quad$ constantes de tiempo

$t_{\mathrm{m}} \quad$ tiempo muerto aparente

$\omega_{\mathrm{n}} \quad$ frecuencia natural

$\zeta$ razón de amortiguamiento

$s \quad$ variable compleja

$\Delta y \quad$ cambio en la señal de salida

$\Delta u \quad$ cambio en la señal de entrada

$K_{\mathrm{cu}}, T_{\mathbf{u}} \quad$ parámetros últimos

$y_{\mathrm{p} 1}, y_{\mathrm{p} 2}, y_{\mathrm{m} 1} \quad$ máximos y mínimos de $\mathrm{y}(\mathrm{t})$

$y_{\mathrm{u}} \quad$ valor final de $\mathrm{y}(\mathrm{t})$

$\Delta t \quad$ semiperiodo

$K_{\mathrm{c}} \quad$ ganancia del controlador $\mathrm{P}$

\section{SOBRE EL AUTOR}

\section{Víctor M. Alfaro}

Profesor Asociado, Departamento de Automática, Escuela de Ingeniería Eléctrica, Universidad de Costa Rica

Apartado postal 1122, 2050 San Pedro, San José, Costa Rica

Tel. 234.2494, Fax. 280.6370, correo electrónico: valfaro@pacuare.eie.ucr.ac.cr 